The Journal of Nonlinear $\mathbf{S}$ ciences and Applications http://www.tjnsa.com

\title{
RELATED FIXED POINT THEOREMS IN FUZZY METRIC SPACES
}

\author{
K. P. R. RAO ${ }^{1 *}$, ABDELKRIM ALIOUCHE $^{2}$ AND G. RAVI BABU ${ }^{3}$
}

\begin{abstract}
We prove a related fixed point Theorem for four mappings which are not continuous in four fuzzy metric spaces, one of them is a sequentially compact fuzzy metric space. Our Theorem in the metric version generalizes Theorem 4 of [1]. Finally, We give a fuzzy version of Theorem 3 of [1].
\end{abstract}

\section{INTRODUCTION AND PRELIMINARIES}

The concept of fuzzy sets was introduced initially by Zadeh [11] in 1965. George and Veeramani [4] modified the concept of fuzzy metric space introduced by Kramosil and Michalek [6]. Recently, Fisher [3], Telci [10] and Aliouche and Fisher [1] proved some related fixed point Theorems in compact metric spaces. Motivated by a work due to Popa [7], we have observed that proving fixed point theorems using an implicit relation is a good idea since it covers several contractive conditions rather than one contractive condition. In this paper, we mainly prove a related fixed point Theorem for four mappings which are not necessarily continuous in four fuzzy metric spaces, using an implicit relation, one of them is a sequentially compact fuzzy metric space. One of our Theorems in the metric version generalizes a theorem of Aliouche and Fisher [1]. We give also a fuzzy version of Theorem 3 of [1]..

Definition $1.1([9])$. A binary operation $*:[0,1] \times[0,1] \longrightarrow[0,1]$ is a continuous $t$-norm if it satisfies the following conditions:

1) $*$ is associative and commutative,

2) $*$ is continuous,

Date: Received: March 2008 ; Revised: Dec 2008

* Corresponding author.

2000 Mathematics Subject Classification. Primary 47H10; Secondary 54H25.

Key words and phrases. Fuzzy metric space, implicit relation, sequentially compact Fuzzy metric space, related fixed point. 
3) $a * 1=a$ for all $a \in[0,1]$,

4) $a * b \leq c * d$ whenever $a \leq c$ and $b \leq d$, for each $a, b, c, d \in[0,1]$.

Two typical examples of a continuous $t$-norm are $a * b=a b$ and $a * b=$ $\min \{a, b\}$.

Definition $1.2([4])$. A 3-tuple $(X, M, *)$ is called a fuzzy metric space if $X$ is an arbitrary (non-empty) set, $*$ is a continuous $t-$ norm a and $M$ is a fuzzy set on $X^{2} \times(0, \infty)$ satisfying the following conditions for each $x, y, z \in X$ and $t, s>0$,

1) $M(x, y, t)>0$,

2) $M(x, y, t)=1$ if and only if $x=y$,

3) $M(x, y, t)=M(y, x, t)$,

4) $M(x, y, t) * M(y, z, s) \leq M(x, z, t+s)$,

5) $M(x, y,):.(0, \infty) \longrightarrow[0,1]$ is continuous.

Definition $1.3([3])$. Let $(X, M, *)$ be a fuzzy metric space.

1) For $t>0$, the open ball $B(x, r, t)$ with center $x \in X$ and radius $0<r<1$ is defined by

$$
B(x, r, t)=\{y \in X: M(x, y, t)>1-r\} .
$$

2) Let $(X, M, *)$ be a fuzzy metric space and $\tau$ be the set of all $A \subset X$ with $x \in A$ if and only if there exist $t>0$ and $0<r<1$ such that $B(x, r, t) \subset A$. Then, $\tau$ is a topology on $X$ induced by the fuzzy metric $M$.

3) A sequence $\left\{x_{n}\right\}$ in $X$ converges to $x$ if and only if for any $0<\epsilon<1$ and $t>0$, there exists $n_{0} \in \mathbb{N}$ such that for all $n \geq n_{0}, M\left(x_{n}, x, t\right)>1-\epsilon$; i.e., $M\left(x_{n}, x_{m}, t\right) \rightarrow 1$ as $n \rightarrow \infty$ for all $t>0$.

4) A sequence $\left\{x_{n}\right\}$ in $X$ is called a Cauchy sequence if and only if for any $0<$ $\epsilon<1$ and $t>0$, there exists $n_{0} \in \mathbb{N}$ such that for all $n, m \geq n_{0}, M\left(x_{n}, x_{m}, t\right)>$ $1-\epsilon$; i.e., $M\left(x_{n}, x_{m}, t\right) \rightarrow 1$ as $n, m \rightarrow \infty$ for all $t>0$.

$5)$ A fuzzy metric space $(X, M, t)$ in which every Cauchy sequence is convergent is said to be complete.

Definition 1.4. A subset $A$ of $X$ is said to be $F$-bounded if there exists $t>0$ and $0<r<1$ such that $M(x, y, t)>1-r$ for all $x, y \in A$.

Lemma $1.5([5])$. Let $(X, M, *)$ be a fuzzy metric space. Then, $M(x, y, t)$ is non-decreasing with respect to $t$, for all $x, y$ in $X$.

Lemma $1.6([5])$. Let $(X, M, *)$ be a fuzzy metric space. Then, $M$ is a continuous function on $X^{2} \times(0, \infty)$.

Definition 1.7. $(X, M, *)$ is said to be sequentially compact fuzzy metric space if every sequence in $X$ has a convergent sub-sequence in it.

Let $\Phi$ be the set of all functions $\phi:[0,1]^{6} \longrightarrow[0,1]$ such that if either $\phi(u, 1, u, v, v, 1)>0$ or $\phi(u, u, 1, v, 1, v)>0$ for all $u, v \in[0,1)$, then $u>v$.

Example 1.8. Let $\phi\left(t_{1}, t_{2}, t_{3}, t_{4}, t_{5}, t_{6}\right)=t_{1}-\min \left\{t_{2}, t_{3}, t_{4}, t_{5}, t_{6}\right\}$. Then $\phi \in \Phi$. 


\section{MAin RESUlts}

Theorem 2.1. Let $\left(X, M_{1}, \theta_{1}\right),\left(Y, M_{2}, \theta_{2}\right),\left(Z, M_{3}, \theta_{3}\right)$ and $\left(W, M_{4}, \theta_{4}\right)$ be fuzzy metric spaces and $B: X \longrightarrow Y, T: Y \longrightarrow Z, A: Z \longrightarrow W, S: W \longrightarrow X$ be mappings satisfying

$$
\text { (1) } \phi_{1}\left(\begin{array}{c}
M_{1}(S A T y, S A T B x, t), M_{1}(x, S A T y, t), \\
M_{1}(x, S A T B x, t), M_{2}(y, B x, t), \\
M_{2}(y, B S A T y, t), M_{2}(B x, B S A T y, t)
\end{array}\right)>0
$$

for all $x \in X, y \in Y$ with $y \neq B x$ and for all $t>0$, where $\phi_{1} \in \Phi$,

$$
\text { (2) } \phi_{2}\left(\begin{array}{c}
M_{2}(B S A z, B S A T y, t), M_{2}(y, B S A z, t), \\
M_{2}(y, B S A T y, t), M_{3}(z, T y, t), \\
M_{3}(z, T B S A z, t), M_{3}(T y, T B S A z, t)
\end{array}\right)>0
$$

for all $z \in Z, y \in Y$ with $z \neq T y$ and for all $t>0$, where $\phi_{2} \in \Phi$,

$$
\text { (3) } \phi_{3}\left(\begin{array}{c}
M_{3}(T B S w, T B S A z, t), M_{3}(z, T B S w, t), \\
M_{3}(z, T B S A z, t), M_{4}(w, A z, t), \\
M_{4}(w, A T B S w, t), M_{4}(A z, A T B S w, t)
\end{array}\right)>0
$$

for all $z \in Z, w \in W$ with $w \neq A z$ and for all $t>0$, where $\phi_{3} \in \Phi$,

$$
\text { (4) } \phi_{4}\left(\begin{array}{c}
M_{4}(A T B x, A T B S w, t), M_{4}(w, A T B x, t), \\
M_{4}(w, A T B S w, t), M_{1}(x, S w, t), \\
M_{1}(x, S A T B x, t), M_{1}(S w, S A T B x, t)
\end{array}\right)>0
$$

for all $x \in X, w \in W$ with $x \neq S w$ and for all $t>0$, where $\phi_{4} \in \Phi$.

Further, suppose that one of the following is true:

(a) $\left(X, M_{1}, \theta_{1}\right)$ is sequentially compact and $S A T B$ is continuous on $X$.

(b) $\left(Y, M_{2}, \theta_{2}\right)$ is sequentially compact and $B S A T$ is continuous on $Y$.

(c) $\left(Z, M_{3}, \theta_{3}\right)$ is sequentially compact and TBSA is continuous on $Z$.

(d) $\left(W, M_{4}, \theta_{4}\right)$ is sequentially compact and ATBS is continuous on $W$.

Then, $S A T B$ has a unique fixed point $u \in X, B S A T$ has a unique fixed point $v \in Y, T B S A$ has a unique fixed point $w \in Z$ and $A T B S$ has a unique fixed point $q \in W$. Further, $B u=v, T v=w, A w=q$ and $S q=u$.

Proof. Suppose that $(a)$ holds. For every $t>0$, define $\phi(x)=M_{1}(x, S A T B x, t)$ for all $x \in X$. Then, there exists $p \in X$ such that $\phi(p)=M_{1}(p, S A T B p, t)=$ $\max \{\phi(x): x \in X\}$.

Suppose that $B S A T B S A T B p \neq B S A T B S A T B S A T B p$. Then, TBSATBp $\neq$ $T B S A T B S A T B p, A T B p \neq A T B S A T B p$ and $p \neq S A T B p$.

Putting $y=B S A T B S A T B p$ and $x=S A T B S A T B S A T B p$ in (1) we have

$$
\phi_{1}\left(\begin{array}{c}
M_{1}(S A T B S A T B S A T B p, S A T B S A T B S A T B S A T B p, t), \\
M_{1}(S A T B S A T B S A T B p, S A T B S A T B S A T B p, t), \\
M_{1}(S A T B S A T B S A T B p, S A T B S A T B S A T B S A T B p, t), \\
M_{2}(B S A T B S A T B p, B S A T B S A T B S A T B p, t), \\
M_{2}(B S A T B S A T B p, B S A T B S A T B S A T B p, t), \\
M_{2}(B S A T B S A T B S A T B p, B S A T B S A T B S A T B p, t)
\end{array}\right)>0
$$


and so

(5) $\phi(S A T B S A T B S A T B p)>M_{2}(B S A T B S A T B p, B S A T B S A T B S A T B p, t)$.

Putting $y=B S A T B S A T B p$ and $z=T B S A T B p$ in (2) we get

$$
\phi_{2}\left(\begin{array}{c}
M_{2}(B S A T B S A T B p, B S A T B S A T B S A T B p, t), \\
M_{2}(B S A T B S A T B p, B S A T B S A T B p, t), \\
M_{2}(B S A T B S A T B p, B S A T B S A T B S A T B p, t), \\
M_{3}(T B S A T B p, T B S A T B S A T B p, t), \\
M_{3}(T B S A T B p, T B S A T B S A T B p, t), \\
M_{3}(T B S A T B S A T B p, T B S A T B S A T B p, t)
\end{array}\right)>0 .
$$

Therefore

(6) $\quad M_{2}(B S A T B S A T B p, B S A T B S A T B S A T B p, t)$

$$
>M_{3}(T B S A T B p, T B S A T B S A T B p, t) .
$$

Putting $z=T B S A T B p$ and $w=A T B p$ in (3) we obtain

$$
\phi_{3}\left(\begin{array}{c}
M_{3}(T B S A T B p, T B S A T B S A T B p, t), \\
M_{3}(T B S A T B p, T B S A T B p, t), \\
M_{3}(T B S A T B p, T B S A T B S A T B p, t), \\
M_{4}(A T B p, A T B S A T B p, t), \\
M_{4}(A T B p, A T B S A T B p, t), \\
M_{4}(A T B S A T B p, A T B S A T B p, t)
\end{array}\right)>0
$$

and so

(7) $M_{3}(T B S A T B p, T B S A T B S A T B p, t)>M_{4}(A T B p, A T B S A T B p, t)$.

Putting $w=A T B p$ and $x=p$ in (4) we have

$$
\phi_{4}\left(\begin{array}{c}
M_{4}(A T B p, A T B S A T B p, t), M_{4}(A T B p, A T B p, t), \\
M_{4}(A T B P, A T B S A T B p, t), M_{1}(p, S A T B p, t), \\
M_{1}(p, S A T B p, t), M_{1}(S A T B p, S A T B p, t)
\end{array}\right)>0 .
$$

Hence

(8) $M_{4}(A T B p, A T B S A T B p, t)>M_{1}(p, S A T B p, t)=\phi(p)$.

From (5), (6), (7) and (8) we get $\phi(S A T B S A T B S A T B p)>\phi(p)$ which is a contradiction. Therefore

(9) $B S A T B S A T B p=B S A T B S A T B S A T B p$.

Denote $B S A T B S A T B p=v \in Y$. Then from (9), $v=B S A T v$.

Let $T v=w \in Z, A w=q \in W, S q=u \in X$. Then $v=B S A T v=B S A w=$ $B S q=B u$.

Also, $S A T B u=S A T v=S A w=S q=u, T B S A w=T B S q=T B u=T v=w$ and $A T B S q=A T B u=A T v=A w=q$.

For the uniqueness of $u$, suppose that $S A T B u^{\prime}=u^{\prime}$ with $u \neq u^{\prime}$. Then, $S A T B u \neq S A T B u^{\prime}, A T B u \neq A T B u^{\prime}, T B u \neq T B u^{\prime}$ and $B u \neq B u^{\prime}$. 
Putting $x=u$ and $y=B u^{\prime}$ in (1) we have

$$
\phi_{1}\left(\begin{array}{c}
M_{1}\left(S A T B u^{\prime}, S A T B u, t\right), M_{1}\left(u, S A T B u^{\prime}, t\right), \\
M_{1}(u, S A T B u, t), M_{2}\left(B u^{\prime}, B u, t\right), \\
M_{2}\left(B u^{\prime}, B S A T B u^{\prime}, t\right), M_{2}\left(B u, B S A T B u^{\prime}, t\right)
\end{array}\right)>0
$$

and so

$$
M_{1}\left(u, u^{\prime}, t\right)>M_{2}\left(B u, B u^{\prime}, t\right) \ldots(10) .
$$

Putting $z=T B u, y=B u^{\prime}$ in (2) we get

$$
\phi_{2}\left(\begin{array}{c}
M_{2}\left(B S A T B u, B S A T B u^{\prime}, t\right), M_{2}\left(B u^{\prime}, B S A T B u, t\right), \\
M_{2}\left(B u^{\prime}, B S A T B u^{\prime}, t\right), M_{3}\left(T B u, T B u^{\prime}, t\right), \\
M_{3}(T B u, T B S A T B u, t), M_{3}\left(T B u^{\prime}, T B S A T B u, t\right)
\end{array}\right)>0 .
$$

Therefore

$$
M_{2}\left(B u, B u^{\prime}, t\right)>M_{3}\left(T B u, T B u^{\prime}, t\right) \ldots . .(11) .
$$

Putting $z=T B u, w=A T B u^{\prime}$ in (3) we obtain

$$
\phi_{3}\left(\begin{array}{c}
M_{3}\left(T B S A T B u^{\prime}, T B S A T B u, t\right), M_{3}\left(T B u, T B S A T B u^{\prime}, t\right), \\
M_{3}(T B u, T B S A T B u, t), M_{4}\left(A T B u^{\prime}, A T B u, t\right), \\
M_{4}\left(A T B u^{\prime}, A T B S A T B u^{\prime}, t\right), M_{4}\left(A T B u, A T B S A T B u^{\prime}, t\right)
\end{array}\right)>0 .
$$

Hence

$$
M_{3}\left(T B u, T B u^{\prime}, t\right)>M_{4}\left(A T B u, A T B u^{\prime}, t\right) \ldots
$$

Putting $x=S A T B u, w=A T B u^{\prime}$ in (4) we have

$$
\phi_{4}\left(\begin{array}{c}
M_{4}\left(A T B S A T B u, A T B S A T B u^{\prime}, t\right), M_{4}\left(A T B u^{\prime}, A T B S A T B u, t\right), \\
M_{4}\left(A T B u^{\prime}, A T B S A T B u^{\prime}, t\right), M_{1}\left(S A T B u, S A T B u^{\prime}, t\right), \\
\left.M_{1}(S A T B u, S A T B S A T B u, t), M_{1}\left(S A T B u^{\prime}, S A T B S A T B u, t\right)\right)
\end{array}\right)>0
$$

and so

$$
M_{4}\left(A T B u, A T B u^{\prime}, t\right)>M_{1}\left(u, u^{\prime}, t\right) . .
$$

Using (10), (11), (12) and (13) we get

$$
M_{1}\left(u, u^{\prime}, t\right)>M_{1}\left(u, u^{\prime}, t\right)
$$

which is a contradiction. Hence, $u$ is the unique fixed point of $S A T B$. Similarly, we can prove the uniqueness of fixed points of $B S A T, T B S A$ and $A T B S$. In a similar manner, the Theorem holds if either (b) or (c) or (d) is true.

The following Example illustrates Theorem 2.1.

Example 2.2. Let $X=[0,1], Y=[1,2), Z=(2,3]$ and $W=[3,4)$ and $M_{1}(x, y, t)=\frac{t}{t+|x-y|}, M_{2}(y, z, t)=\frac{t}{t+|y-z|}, M_{3}(z, w, t)=\frac{t}{t+|z-w|}$ and $M_{4}(w, x, t)=\frac{t}{t+|w-x|}$.

Define $B: X \longrightarrow Y$ by:

$$
B x=\left\{\begin{array}{cl}
1 & \text { if } x \in[0,3 / 4] \\
3 / 2 & \text { if } x \in(3 / 4,1]
\end{array}\right.
$$


$T: Y \longrightarrow Z$ by $T y=3$ for all $y \in Y, A: Z \longrightarrow W$ by

$$
A z=\left\{\begin{array}{cl}
7 / 2 & \text { if } x \in(2,5 / 2] \\
3 & \text { if } x \in(5 / 2,3]
\end{array}\right.
$$

and $S: W \longrightarrow X$ by $S w=1$ for all $w \in W$. Let

$$
\begin{aligned}
\phi_{1}\left(t_{1}, t_{2}, t_{3}, t_{4}, t_{5}, t_{6}\right) & =t_{1}-\min \left\{t_{2}, t_{3}, t_{4}, t_{5}, t_{6}\right\} \text { and } \\
\phi_{1} & =\phi_{2}=\phi_{3}=\phi_{4} .
\end{aligned}
$$

In this Example, the inequalities (1), (2), (3) and (4) are satisfied since the value of the left hand side of each inequality is 1 .

Clearly, $S A T B(1)=1, B S A T(3 / 2)=3 / 2, T B S A(3)=3, A T B S(3)=3$ and $B 1=3 / 2, T(3 / 2)=3, A 3=3, S 3=1$.

If $B=T, T=S, A=R, S=I$ (Identity map) and $W=X$ in Theorem 2.1 we get the following Theorem.

Theorem 2.3. Let $\left(X, M_{1}, \theta_{1}\right),\left(Y, M_{2}, \theta_{2}\right)$ and $\left(Z, M_{3}, \theta_{3}\right)$ be fuzzy metric spaces and $T: X \longrightarrow Y, S: Y \longrightarrow Z, R: Z \longrightarrow X$ be mappings satisfying

(1) $\phi_{1}\left(\begin{array}{c}M_{1}(R S y, R S T x, t), M_{1}(x, R S y, t), M_{1}(x, R S T x, t), \\ M_{2}(y, T x, t), M_{2}(y, T R S y, t), M_{2}(T x, T R S y, t)\end{array}\right)>0$ for all $x \in X, y \in Y$ with $y \neq T x$ and for all $t>0$, where $\phi_{1} \in \Phi$,

(2) $\phi_{2}\left(\begin{array}{c}M_{2}(T R z, T R S y, t), M_{2}(y, T R z, t), M_{2}(y, T R S y, t), \\ M_{3}(z, S y, t), M_{3}(z, S T R z, t), M_{3}(S y, S T R z, t)\end{array}\right)>0$ for all $z \in Z, y \in Y$ with $z \neq S y$ and for all $t>0$, where $\phi_{2} \in \Phi$,

(3) $\phi_{3}\left(\begin{array}{c}M_{3}(S T x, S T R z, t), M_{3}(z, S T x, t), M_{3}(z, S T R z, t), \\ M_{1}(x, R z, t), M_{1}(x, R S T x, t), M_{1}(R z, R S T x, t)\end{array}\right)>0$

for all $z \in Z, x \in X$ with $x \neq R z$ and for all $t>0$, where $\phi_{3} \in \Phi$. Further, suppose that one of the following is true:

(a) $\left(X, M_{1}, \theta_{1}\right)$ is sequentially compact and $R S T$ is continuous on $X$.

(b) $\left(Y, M_{2}, \theta_{2}\right)$ is sequentially compact and TRS is continuous on $Y$.

(c) $\left(Z, M_{3}, \theta_{3}\right)$ is sequentially compact and $S T R$ is continuous on $Z$.

Then, RST has a unique fixed point $u \in X, T R S$ has a unique fixed point $v \in Y$ and $S T R$ has a unique fixed point $w \in Z$. Further, $T u=v, S v=w$ and $R w=u$.

If $R=I$ (Identity map) and $Z=X$ in Theorem 2.3 we obtain

Theorem 2.4. Let $\left(X, M_{1}, \theta_{1}\right)$ and $\left(Y, M_{2}, \theta_{2}\right)$ be fuzzy metric spaces and $T$ : $X \longrightarrow Y, S: Y \longrightarrow X$ be mappings satisfying

(1) $\phi_{1}\left(\begin{array}{c}M_{1}(S y, S T x, t), M_{1}(x, S y, t), M_{1}(x, S T x, t), \\ M_{2}(y, T x, t), M_{2}(y, T S y, t), M_{2}(T x, T S y, t)\end{array}\right)>0$

for all $x \in X, y \in Y$ with $y \neq T x$ and for all $t>0$, where $\phi_{1} \in \Phi$,

(2) $\phi_{2}\left(\begin{array}{c}M_{2}(T x, T S y, t), M_{2}(y, T x, t), M_{2}(y, T S y, t), \\ M_{1}(x, S y, t), M_{1}(x, S T x, t), M_{1}(S y, S T x, t)\end{array}\right)>0$

for all $x \in X, y \in Y$ with $x \neq S y$ and for all $t>0$, where $\phi_{2} \in \Phi$. Further, suppose that one of the following is true: 
(a) $\left(X, M_{1}, \theta_{1}\right)$ is sequentially compact and $S T$ is continuous on $X$.

(b) $\left(Y, M_{2}, \theta_{2}\right)$ is sequentially compact and TS is continuous on $Y$.

Then, $S T$ has a unique fixed point $u \in X$ and $T S$ has a unique fixed point $v \in Y$.

Further, $T u=v$ and $S v=u$.

1) The metric version of Theorem 2.4 in compact metric spaces generalizes and improves Theorem 4 of Aliouche and Fisher [1] under the implicit relation $\phi: \mathbb{R}_{+}^{6} \rightarrow \mathbb{R}$ such that $\phi(u, u, 0, v, 0, v)<0$ or $\phi(u, 0, u, v, v, 0)<0$ implies $u<v$.

2) If $\phi_{1}\left(t_{1}, t_{2}, t_{3}, t_{4}, t_{5}, t_{6}\right)=\phi_{2}\left(t_{1}, t_{2}, t_{3}, t_{4}, t_{5}, t_{6}\right)=t_{1}-\min \left\{t_{2}, t_{3}, t_{4}, t_{5}, t_{6}\right\}$ in Theorem 2.4, we get a fuzzy version of a Theorem of Fisher [3].

Finally, we give a fuzzy version of Theorem 3 of Aliouche and Fisher [1] using the following implicit relations.

We denote by $\Psi$ the set of all functions $\psi:[0,1]^{4} \longrightarrow[0,1]$ such that

:(i) $\psi$ is upper semi continuous in each coordinate variable,

(ii) $\psi$ is decreasing in $3 \mathrm{rd}$ and 4 th variable,

(iii) if either $\psi(u, v, 1, u) \geq 0$ or $\psi(u, 1, v, 1) \geq 0$ or $\psi(u, v, u, 1) \geq 0$ for all $u, v \in[0,1]$, then $u \geq v$.

Example 2.5. $\psi\left(t_{1}, t_{2}, t_{3}, t_{4}\right)=t_{1}-\min \left\{t_{2}, t_{3}, t_{4}\right\}$,

Example 2.6. $\psi\left(t_{1}, t_{2}, t_{3}, t_{4}\right)=t_{1}-\phi_{1}\left(\min \left\{t_{2}, t_{3}, t_{4}\right\}\right)$, where $\phi_{1}:(0,1] \longrightarrow(0,1]$ is an increasing and continuous function with $\phi(t)>t$ for $0<t<1$. For example $\phi_{1}(t)=\sqrt{t}$ or $\phi_{1}(t)=t^{h}$ for $0<h<1$.

We need the following Lemma of [2].

Lemma $2.7([2])$. Let $\left\{x_{n}\right\}$ be a sequence in a fuzzy metric space $(X, M, *)$ with $M(x, y, t) \longrightarrow 1$ as $t \longrightarrow \infty$ for all $x, y \in X$. If there exists a number $k \in(0,1)$ such that

$$
M\left(x_{n+1}, x_{n}, k t\right) \geq M\left(x_{n}, x_{n-1}, t\right)
$$

for all $t>0$ and $n=1,2,3, \ldots$, then $\left\{x_{n}\right\}$ is a Cauchy sequence in $X$.

Theorem 2.8. Let $\left(X, M_{1}, \theta_{1}\right)$ and $\left(Y, M_{2}, \theta_{2}\right)$ be complete fuzzy metric spaces with $M_{1}\left(x, x^{\prime}, t\right) \longrightarrow 1$ as $t \longrightarrow \infty$ for all $x, x^{\prime} \in X$ and $M_{2}\left(y, y^{\prime}, t\right) \longrightarrow 1$ as $t \longrightarrow \infty$ for all $y, y^{\prime} \in Y$. Let $T: X \longrightarrow Y, S: Y \longrightarrow X$ be mappings satisfying:

(1) $\psi_{1}\left(M_{1}(S y, S T x, k t), M_{2}(y, T x, t), M_{1}(x, S y, t), M_{1}(x, S T x, t)\right) \geq 0$,

(2) $\psi_{2}\left(M_{2}(T x, T S y, k t), M_{1}(x, S y, t), M_{2}(y, T x, t), M_{2}(y, T S y, t)\right) \geq 0$

for all $x \in X, y \in Y$ and for all $t>0$, where $\psi_{1}, \psi_{2} \in \Psi$ and $0<k<1$.

Then, $S T$ has a unique fixed point $u \in X$ and $T S$ has a unique fixed point $v \in Y$. Further, $T u=v$ and $S v=u$.

Proof. Let $x_{0}$ be an arbitrary point in $X$. We define the sequences $\left\{x_{n}\right\}$ and $\left\{y_{n}\right\}$ in $X$ and $Y$ respectively by: $y_{n}=T x_{n-1}, x_{n}=S y_{n}$ for $n=1,2, \ldots$.

Putting $x=x_{n}$ and $y=y_{n}$ in (1), we have

$$
\psi_{1}\left(M_{1}\left(x_{n}, x_{n+1}, k t\right), M_{2}\left(y_{n}, y_{n+1}, t\right), 1, M_{1}\left(x_{n}, x_{n+1}, t\right)\right) \geq 0 .
$$


Since $\psi_{1}$ is decreasing in 4 th variable, we get

$$
\psi_{1}\left(M_{1}\left(x_{n}, x_{n+1}, k t\right), M_{2}\left(y_{n}, y_{n+1}, t\right), 1, M_{1}\left(x_{n}, x_{n+1}, k t\right)\right) \geq 0 .
$$

From (iii), we obtain

$$
M_{1}\left(x_{n}, x_{n+1}, k t\right) \geq M_{2}\left(y_{n}, y_{n+1}, t\right) . .(3
$$

Putting $x=x_{n-1}$ and $y=y_{n}$ in (2), we have

$$
\psi_{2}\left(M_{2}\left(y_{n}, y_{n+1}, k t\right), M_{1}\left(x_{n-1}, x_{n}, t\right), 1, M_{2}\left(y_{n}, y_{n+1}, t\right)\right) \geq 0 \text {. }
$$

As $\psi_{2}$ is decreasing in 4 th variable, we get

$$
\psi_{2}\left(M_{2}\left(y_{n}, y_{n+1}, k t\right), M_{1}\left(x_{n-1}, x_{n}, t\right), 1, M_{2}\left(y_{n}, y_{n+1}, k t\right)\right) \geq 0 \text {. }
$$

From (iii), we obtain

$$
M_{2}\left(y_{n}, y_{n+1}, k t\right) \geq M_{1}\left(x_{n-1}, x_{n}, t\right) \ldots(4) .
$$

Using (3) and (4) we have for $n=1,2, \ldots$.

$$
\begin{aligned}
M_{1}\left(x_{n}, x_{n+1}, t\right) & \geq M_{1}\left(x_{n-1}, x_{n}, t / k^{2}\right) \text { and } \\
M_{2}\left(y_{n}, y_{n+1}, t\right) & \geq M_{2}\left(y_{n-1}, y_{n}, t / k^{2}\right) .
\end{aligned}
$$

From Lemma 2.7, it follows that $\left\{x_{n}\right\}$ and $\left\{y_{n}\right\}$ are Cauchy sequences in $X$ and $Y$ respectively. Hence, $\left\{x_{n}\right\}$ converges to $u \in X$ and $\left\{y_{n}\right\}$ converges to $v \in Y$. Putting $x=x_{n-1}$ and $y=v$ in (1), we get

$\psi_{1}\left(M_{1}\left(S v, S T x_{n-1}, k t\right), M_{2}\left(v, T x_{n-1}, t\right), M_{1}\left(x_{n-1}, S v, t\right), M_{1}\left(x_{n-1}, S T x_{n-1}, t\right)\right) \geq 0$

Letting $n \longrightarrow \infty$, we have

$$
\psi_{1}\left(M_{1}(S v, u, k t), 1, M_{1}(u, S v, t), 1\right) \geq 0 .
$$

Using (iii), we obtain

$$
M_{1}(S v, u, k t) \geq M_{1}(u, S v, t)
$$

and so $S v=u$. Similarly, we can show that $T u=v$. Now, $S T u=S v=u$ and $T S v=T u=v$.

To prove the uniqueness of $u$, suppose that $S T$ has a second fixed point $u^{\prime}$ in $X$.

Putting $x=u^{\prime}, y=v$ in (1), we get

$$
\left.\psi_{1}\left(M_{1}\left(u, u^{\prime}, k t\right), M_{2}\left(T u, T u^{\prime}, t\right), M_{1}\left(u^{\prime}, u, t\right), 1\right)\right) \geq 0 .
$$

Since $\psi_{1}$ is decreasing in 3 rd variable, we have

$$
\left.\psi_{1}\left(M_{1}\left(u, u^{\prime}, k t\right), M_{2}\left(T u, T u^{\prime}, t\right), M_{1}\left(u^{\prime}, u, k t\right), 1\right)\right) \geq 0 .
$$

From (iii), we obtain

$$
M_{1}\left(u, u^{\prime}, k t\right) \geq M_{2}\left(T u, T u^{\prime}, t\right) .
$$

Similarly, we have

$$
M_{2}\left(T u, T u^{\prime}, k t\right) \geq M_{1}\left(u, u^{\prime}, t\right) .
$$

Hence

$$
M_{1}\left(u, u^{\prime}, t\right) \geq M_{1}\left(u, u^{\prime}, t / k^{2}\right)
$$

and so $u=u^{\prime}$. The uniqueness of $v$ follows in a similar manner. 
1) If $\psi_{1}\left(t_{1}, t_{2}, t_{3}, t_{4}\right)=\psi_{2}\left(t_{1}, t_{2}, t_{3}, t_{4}\right)=t_{1}-\min \left\{t_{2}, t_{3}, t_{4}\right\}$ in Theorem 2.7 , we get a fuzzy version of a Theorem of Fisher [3].

2) As in Theorems 2.4 and 2.7, we can obtain fuzzy versions of Theorems of $[9]$.

The following Example support our Theorem 2.7.

Example 2.9. Let $X=[0,1]=Y$ and $M_{1}(x, y, t)=M_{2}(y, x, t)=\frac{t}{t+|x-y|}$ for all $x, y \in X$ and for all $t>0$. Define $T: X \longrightarrow Y$ and $S: Y \longrightarrow X$ by:

$$
T x=\left\{\begin{array}{rr}
x / 2 & \text { if } x \in(0,1], \\
1 / 2 & \text { if } x=0 .
\end{array},\right.
$$

$S y=1 / 2$ for all $y \in Y$. Let

$$
\begin{aligned}
\psi_{1}\left(t_{1}, t_{2}, t_{3}, t_{4}\right) & =\psi_{2}\left(t_{1}, t_{2}, t_{3}, t_{4}\right) \\
& =t_{1}-\min \left\{t_{2}, t_{3}, t_{4}\right\} .
\end{aligned}
$$

In this Example, the inequality (1) is satisfied since the value of the left hand side of inequality is 1 and the inequality (2) is satisfied with $k=1 / 2$.

Clearly, $S T(1 / 2)=1 / 2, T S(1 / 4)=1 / 4, S(1 / 4)=1 / 2$ and $T(1 / 2)=1 / 4$.

\section{REFERENCES}

[1] A. Aliouche and B. Fisher, Fixed point theorems for mappings satisfying implicit relation on two complete and compact metric spaces, Applied Mathematics and Mechanics., 27 (9) (2006), 1217-1222.

[2] Y. J. Cho, Fixed points in fuzzy metric spaces, J. Fuzzy. Math., 5 (4) (1997), 949-962.

[3] B. Fisher, Fixed point on two metric spaces, Glasnik Mat., 16 (36) (1981), 333-337.

[4] A. George and P. Veeramani, On some result in fuzzy metric space, Fuzzy Sets Syst., 64 (1994), 395-399.

[5] M. Grabiec, Fixed points in fuzzy metric spaces Fuzzy Sets Syst., 27 (1988), 385-389.

[6] I. Kramosil and J. Michalek, Fuzzy metric and statistical metric spaces, Kybernetica., 11 (1975), 326-334.

[7] V. Popa, Some fixed point theorems for compatible mappings satisfying an implicit relation, Demonstratio Math., 32 (1999),157-163.

[8] J. Rodríguez López and S. Ramaguera, The Hausdorff fuzzy metric on compact sets, Fuzzy Sets Sys., 147 (2004), 273-283.

[9] B. Schweizer and A. Sklar, Statistical metric spaces, Pacific J. Math. 10 (1960), 313-334.

[10] M.Telci, Fixed points on two complete and compact metric spaces, Applied Mathematics and Mechanics., 22 (5) (2001), 564-568.

[11] L. A. Zadeh, Fuzzy sets, Inform and Control., 8 (1965), 338-353.

${ }^{1}$ and ${ }^{3}$ Dept. Of Applied Mathematics, Acharya Nagarjuna, University-Nuzvid Campus, NUZVID-521 201, Krishna Dt., A.P., INDIA.

E-mail address: kprrao2004@yahoo.com

2 Department of Mathematics, University of Larbi Ben M' Hidi, Oum-El-Bouaghi, 04000, Algeria.

E-mail address: alioumath@yahoo.fr 